Helgoländer wiss. Meeresunters. 25, 85-92 (1973)

\title{
Über die Entstehung des Nesselschlauches der Nematocysten von Dipurena reesi (Hydrozoa)
}

\author{
H. GÜNZL \\ Zoologisches Institut der Universität Tübingen; \\ Tübingen, Bundesrepublik Deutschland
}

\begin{abstract}
On the formation of the stinging tube in the nematocysts of Dipurena reesi (Hydrozoa). The differentiation of the stinging tube of a stenotele was studied by light microscopy in the hydroid Dipurena reesi VAnNuccr. It was found, that the central body of the matrix cares for the correct position of the thread in the capsule, and that thereafter the stylets are formed from its material. The stylets and bristles of the shaft arise along a PJA-positive axial thread. The wall of the shatt grows down from the apical pole of the capsule, and envelops the primordia of the stylets and bristles.
\end{abstract}

\section{EINLEITUNG}

Seit MöBIus (1866) zum ersten Mal die Entwicklung der Nesselkapseln der Cnidarier genauer untersucht hat, sind zahlreiche Arbeiten erschienen, welche sich mit diesem komplizierten Sekretionsvorgang beschäftigen. Trotzdem konnten bis heute viele Einzelheiten der Cnidogenese noch nicht geklärt werden. Besonders schwierig ist die Analyse der Differenzierung des Nesselschlauches mit seinen Borsten, Dornen und Stiletten. Ist der Außenschlauch die Anlage des Nesselschlauches, wie Jrckeli (1883), I wanzoff (1896), Schneider(1900), Slaut'Terback \& Fa wCETt (1959) u. a. annehmen, oder wird der Nesselschlauch de novo in der Kapselanlage gebildet, wie MöвIUs (1866), Will (1910), Ewald (1915), Chapman \& Tilney (1959) u. a. meinen? Die elektronenmikroskopische Untersuchung von WESTFALL (1966) läßt zwar kaum noch einen $Z$ weifel darüber, daß der Nesselschlauch in dem Sekret gebildet wird, welches Außenschlauch und Kapselanlage ausfüllt. Doch damit ergeben sich neue Probleme: Spielt dabei der Außenschlauch eine Rolle? Woher kommt das Material für Wand und Bewehrung des Nesselschlauches, und wie können die doch recht komplizierten Strukturen im Kapselsekret entstehen?

Wenig Beachtung wurde in diesem Zusammenhang bisher der Bedeutung eines zentralen Selkretstrànges gewidmet, welcher sich durch sein färberisches Verhalten deutlich vom tubrigen 'Thhalt der Kapselanlage unterscheidet. IwANZOFF (1896) deutete ihín wegen seiner oft spiraligen Form als Anlage des Nesselfadens. ScHNEIDER (1900) war dagegen der Meinung, daß es sich um cine Vorstufe des:Nesselgiftes der reifen Kapsel 
handelt. Will (1910) und EWALD (1915) fanden dann, daß der Kapselinhalt aus zwei Sekreten gebildet wird, wobei sich das zentrale Sekret im peripheren auflöst.

In dieser Arbeit wird nun die Differenzierung des Nesselschlauches mit histochemischen und autoradiographischen Methoden erneut untersucht. Dabei steht die Bedeutung des Zentralsekrets für diesen Differenzierungsvorgang im Vordergrund.

\section{MATERIAL UND METHODIK}

Die Untersuchungen wurden an den Polypenkolonien von Dipurena reesi VAN-

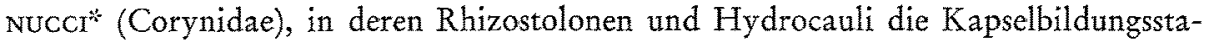

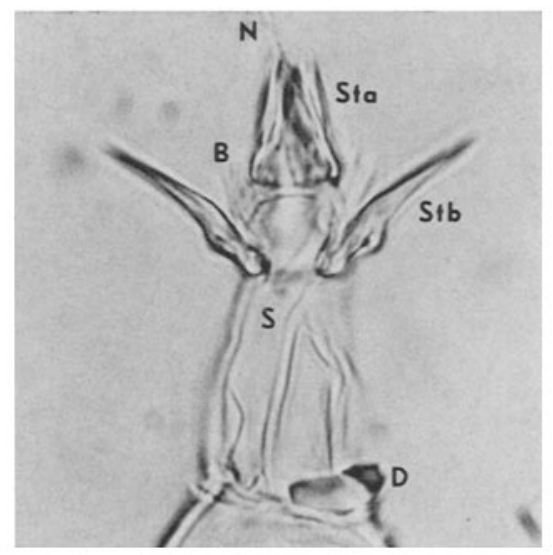

Abb. 1: Dipurena reesi. Explodierte Nesselkapsel. Der Nesselschlauch besteht aus dem Schaft (S), an welchem drei apikale Stilette (Sta), drei basale Stilette (Stb) und die in drei Reihen angeordneten Borsten (B) inserieren und dem Nesselfaden (N). D Kapseldeckel. (Quetschpräparat; Hellfeld; $1500: 1$ )

dien anzutreffen sind, durchgeführt. Der Hydroidpolyp besitzt nur Stenotelen, die in zwei Größen vorkommen. Hier werden lediglich die Anlagen des großen Kapseltyps berücksichtigt, dessen Nesselschlauch Abbildung 1 zeigt. (Nähere Einzelheiten über Herkunft und Kultur des Versuchstieres vgl. GünzL 1964 und 1968.)

Es wurden Paraffinschnitte $(2,5-5 \mu \mathrm{m})$ von Sublimat-Formol-fixiertem Material hergestellt und diese nach der Perjodsäure-Schiff-Methode (Spannhor 1967), mit Orcein nach Taenzer-Unna (Romers 1948) oder mit 0,1\% Trypanblau in 0,2 $\mathrm{m}$ Phosphat$0,1 \mathrm{~m}$ Citratpuffer bei pH 5,8 gefärbt. Zum Vergleich dienten Quetschpräparate von unfixiertem Stologewebe.

Die radioaktive Markierung erfolgte mit $\mathrm{H} 3$-Histidin (L-Histidine-2,5- $\mathrm{H} 3$, $1 \mathrm{mCi} / \mathrm{ml})$. Die Aminosäure wurde dem Artemienzuchtwasser zugesetzt $(0,05 \mathrm{ml} \mathrm{zu}$ $10 \mathrm{ml}$ Seewasser) und die Nauplien drei Tage in dieser Lösung bei $20^{\circ} \mathrm{C}$ gehalten. Dann wurden sie an vorher isolierte Kolonieteile verfüttert. Die Zuchttemperatur die-

* Neuerdings Sarsia reesi (BRnKmann-Voss 1970). 
ser Kolonien betrug ebenfalls $20^{\circ} \mathrm{C}$. Nach ein bzw. drei Tagen erfolgte die Fixierung der Kolonien in Sublimat-Formol. Zur Färbung der Schnitte diente die DiazoniumsalzKupplung (Spannhof 1967). Die Beschichtung wurde mit AR 10 stripping film durchgeführt; die Belichtungszeit betrug 14 Tage.

\section{BEFUNDE}

Während der Sekretionsphase werden von den Cnidoblasten - wie bekannt - drei Stoffe oder Stoffgruppen gebildet (Abb. 2): Zentralsekret, Peripherer Kapselinhalt und Wandsekret. Unser Augenmerk soll sich in dieser Arbeit nur auf die beiden zuerst genannten Sekrete richten. Diese gelangen während der frühen Sekretionsphase aus dem Golgiapparat direkt in die Kapselanlage. Später werden Golgiapparat und Kapselanlage durch den Außenschlauch verbunden, welcher sich bis zum Ende der Sekretionsphase stark verlängert (vgl. StautTERBACK \& FAw CETT 1959). Das kapselseitige Ende dieses Schlauches ist kegelförmig erweitert und bildet so ein Ubergangsstück zwischen dem dünnen Schlauch und der wesentlich größeren Offnung der Kapselanlage (Abb. 2c)*.

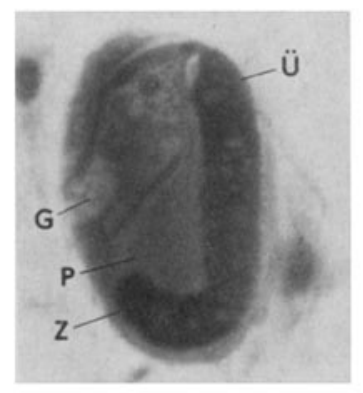

a

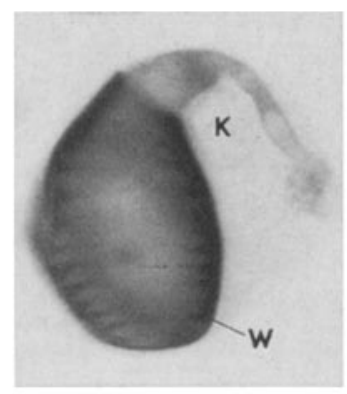

b

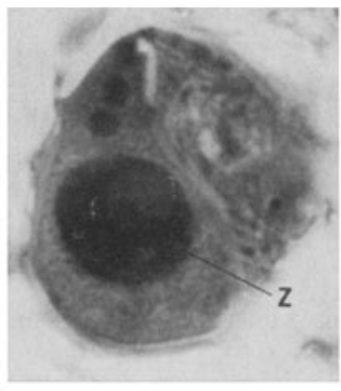

C

Abb. 2: Dipurena reesi. Kapselanlagen mit Außenschlauch. $a$ und $b$ Mittlere Sekretionsphase. G Golgiapparat; K Zellkern; P Peripherer Kapselinhalt; U Ubergangsstück; W Kapselwand mit gefalteter, später jedoch glatter Innenfläche; Z Zentralsekret. $c$ Späte Sekretionsphase. Der lange Außenschlauch bildet im Plasma einen Knäuel. ( $a$ und $c$ Trypanblau, $b$ Orcein; Grünfilter; $1500: 1)$

Das Zentralsekret zeichnet sich durch seine starke Acidophilie aus, welche auf einen hohen Gehalt an Histidin zurüdkzuführen ist (positiver Histidintest, vgl. GüNZz 1968). Es färbt sich daher mit Trypanblau bei pH 5,8 dunkel an (Abb. 2a, c). Während der frühen Sekretionsphase durchzieht das Sekret die Kapselanlage in Form eines Stranges (Abb. 2a). Später rundet es sich $a b$ und verliert den Zusammenhang mit dem Zentralsekret des Außenschlauches (Abb. 2c). Der Zuwachs erfolgt dann durch kleine kugelige Sekretportionen, die in der Kapselanlage mit dem Sekretkörper verschmelzen. Am Ende der Sekretionsphase liegt im Zentrum der Kapselanlage entweder eine ein-

* Der helle Hof, welcher den Außenschlauch umgibt, ist nicht immer sichtbar und stellt deshalb wohl ein Fixierungsartefakt dar. 


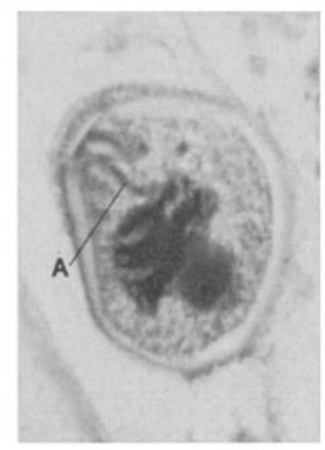

a

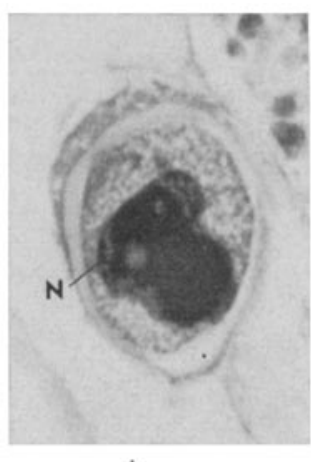

b

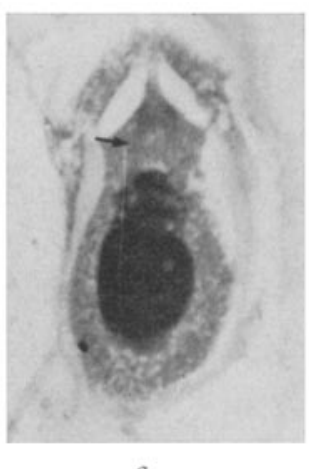

Abb. 3: Dipurena reesi. Kapselanlagen mit zweiteiligem Zentralsekret, auf dessen apikalem Teil der Nesselfaden aufgewickelt ist. $a$ und $b$ Zwei Schnitte durch eine Anlage mit beginnender Schaftdifferenzierung. A Schaftachse; N Nesselfaden. $c$ Frühes Differenzierungsstadium (Ubergangsstïck noch nicht reduziert). Pfeil: Einwandernder Nesselfladen? (Trypanblau; Grunfilter; $1500: 1$ )

heitliche Sekretkugel oder ein aus zwei Kalotten zusammengesetzter Sekretkörper (Abb. 3).

Die Differenzierungsphase beginnt mit dem Erscheinen des Nesselfadens in der Kapselanlage. Er ist an der Peripherie des Sekretkörpers in quer zur Längsachse der Kapsel liegenden Windungen aufgewickelt (Abb. 3, 6a). Diese Lage entspricht der Anordnung des Fadens in der reifen Kapsel. Einen Hinweis auf die Entstehungsweise des Nesselfadens gibt uns die Tatsache, daß bei zweiteiligem Zentralsekret die basale Kalotte immer ganz oder größtenteils fadenfrei bleibt (Abb. 3). Würde man annehmen, daß der Nesselfaden im Zentralsekret entsteht oder dieses seine Bildung im peripheren Kapselinhalt induziert, so wäre der oben genannte Sachverhalt schwer verständlich. Man muß vielmehr annehmen, daß der Nesselfaden außerhalb der Kapselanlage (im Außenschlauch?) gebildet wird und das Zentralsekret lediglich als Spule für den vom Apikalpol der Kapselanlage her einwandernden Faden dient. Diese Funktion beschränkt sich bei zweiteiligem Zentralkörper auf den der Kapselöffnung näher liegenden Teil.

Der nächste Differenzierungsschritt beginnt mit der Auflösung des Zentralsekrets im peripheren Kapselinhalt und der Entstehung der Schaftanlage (Abb. 4a, b, 6). Das früheste lichtmikroskopisch nachweisbare Stadium der Schaftanlage ist ein PJS-positiver, schleifenförmig gebogener Faden am Apikalpol der Kapselanlage außerhalb des Zentralsekrets (Abb. 4a). Untersuchungen an Quetschpräparaten haben gezeigt, daß dieser Faden mit dem Nesselfaden verbunden ist, also dessen basale Fortsetzung darstellt. Wie stark er sich iber sein chemisches Verhalten hinaus auch in seiner Struktur vom Nesselfaden unterscheidet, könnte nicht geklärt werden. Der zentral gelegene Fadenschenkel ist die Schaftachse, auf welcher die Stilett- und Borstenanlagen als zunächst gleich große, acidophile Leisten erscheinen (Abb. 5). Diese Leisten sind it drei Spirallinien angeordnet. Bald fallen aber die Stilettanlagen durch ihr stärkeres Wachstum auf, wodurch die PJS-positive Achse allmählich basalwärts verschoben wird (Abb. 4c). 


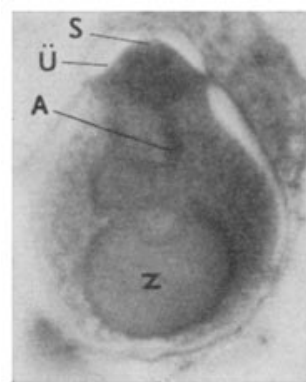

a

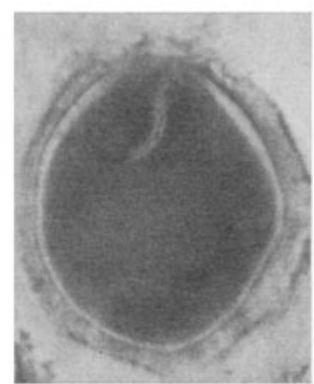

b

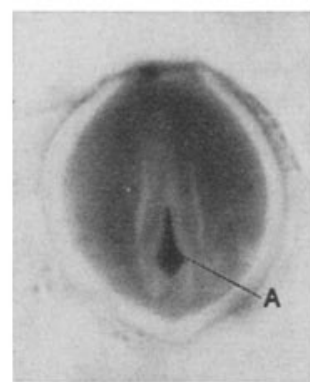

$\mathrm{c}$

Abb. 4: Dipurena reesi. Differenzierung des Schaftes. a Bildung des Achsenfadens (A) und der Schaftwand (S). U Ubergangsstück; $Z$ Zentralsekret. $b$ Etwas älteres Stadium im Phasenkontrast. Die Stilett- und Borstenanlagen auf dem Achsenfaden sind sichtbar. $c$ Kapselanlage mit ausdifferenziertem Schaft. (Perjodsäure-Schiff; Grünfilter; $1500: 1$ )
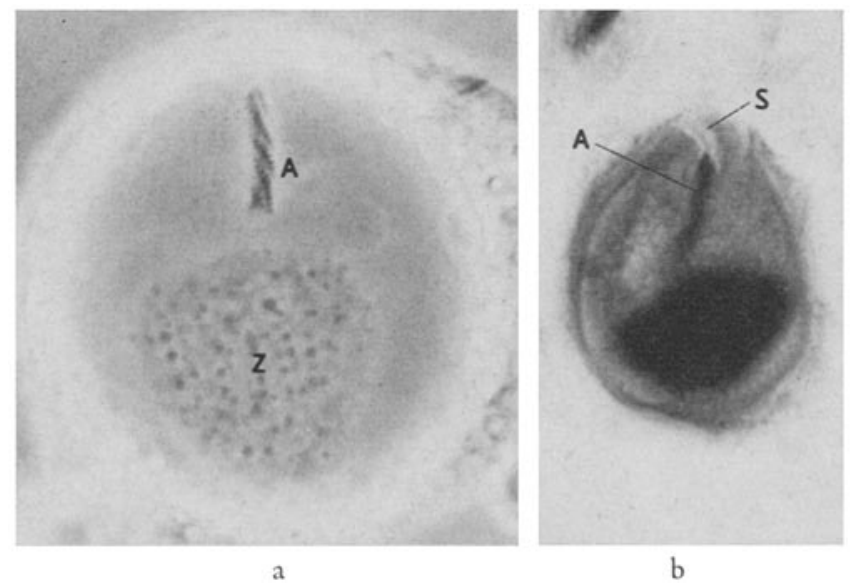

Abb. 5: Dipurena reesi. Etwa gleich alte Stadien mit Schaftanlage. a Unfixierte Kapselanlage im Phasenkontrast. A Schaftachse mit den Stilett- und Borstenanlagen; $Z$ Zentralsekret in Auflösung. $b$ Paraffinschnitt mit Trypanblau gefärbt. S Anlage der Schaftwand. ( $b$ mit Grünfilter; a $3000: 1, b$ 1500:1)

Die Stilett- und Borstenanlagen sind zunächst nicht durch die Schaftwand vom Kapselinhalt getrennt. Die Schaftwand schiebt sich erst allmählich von der apikalen Offnung des Übergangsstückes her (der Außenschlauch ist nach dem Erscheinen des Nesselfadens nicht mehr nachweisbar) über die Schaftachse (Abb. 4a, 5b). Diese Tatsache und das Fehlen des Außenschlauches, der die Sekrete vom Golgiapparat in die Kapselanlage überleitet, sprechen für eine Bildung der Stilette und Borsten aus zuvor in der Kapsel deponiertem Material. Auf die Bedeutung des basischen Zentralsekrets für diesen Differenzierungsvorgang weist die ebenfalls starke Acidophilie der Stilette hin.

Zur Klärung des Zusammenhangs zwischen Zentralsekret und Stilettdifferenzicrung diente die Markierung mit H3-Histidin. Sie hatte folgendes Ergebnis: Einen Tag nach der Verabreichung der Aminosäure ist das Zentralsekret der Sekretionsstadien kräftig markiert (Abb.7a). Frühe Differenzierungsstadien enthalten ebenfalts $\mathrm{H} 3$ - 
Histidin (Abb. $7 \mathrm{~b}$ ), sind jedoch in allen Teilen schwächer markiert, und die Stilettanlagen treten nicht hervor. Altere Stadien (vgl. Abb. 6c) sind nach einem Tag noch nicht markiert. Markierte Kapselanlagen dieses Differenzierungsgrades treten erst in der drei Tage nach der Fütterung fixierten Kolonie auf. Kapselsekret (aus dem Peripheren Kapselinhalt und dem in ihm aufgelösten Zentralsekret bestehend) und Stilette

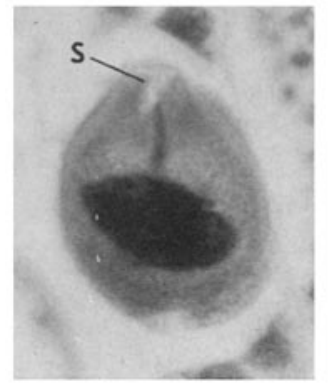

a

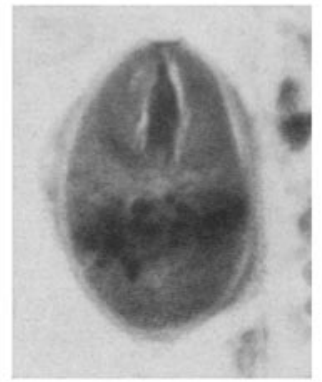

b

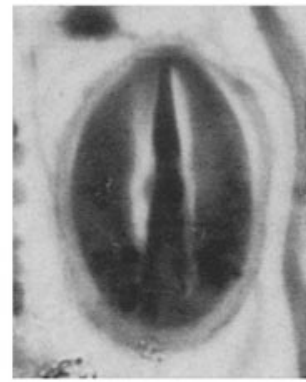

Abb. 6: Dipurena reesi. Die verschieden alten Kapselanlagen zeigen die Auflösung des Zentralsekrets und die Differenzierung des Schaftes. S Anlage der Schaftwand. (Trypanblau; Grünfilter: $1500: 1)$

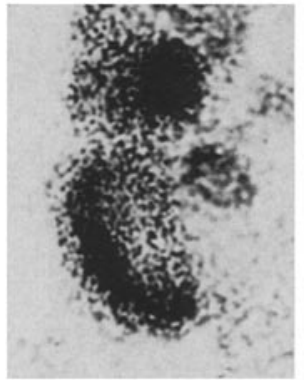

a

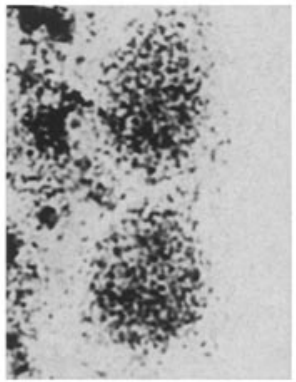

b

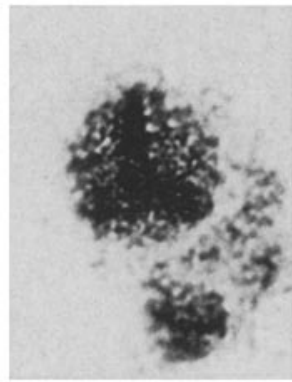

c

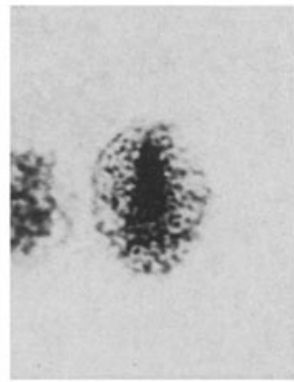

$\mathrm{d}$

Abb. 7: Dipurena reesi. Markierung mit H3-Histidin. Einen Tag nach Fütterung: a Sekretionsstadien mit stark markiertem Zentralsekret. $b$ Zwei Anlagen im frühen Differenzierungsstadium (vgl. Abb. 6b). Drei Tage nach Fütterung: $c$ Frühes Differenzierungsstadium mit stark markierten Stiletten und Zentralsekret. $d$ Spätes Differenzierungsstadium. (Rotfilter; $750: 1)$

sind bei diesen in der Regel stärker markiert als bei den frühen Differenzierungsstadien der Eintagpräparate, und die Stilette zeichnen sich in den Autoradiogrammen oft sehr deutlich durch besonders dichte Körnung ab (Abb. 7d).

Histidin gelangt also während der Sekretionsphase in die Kapselanlage und wird dort im Zentralsekret gespeichert. Späte Sekretionsstadien können nur noch wenig H3Histidin aufnehmen. Sie sind als frühe Reifungsstadien daher schwächer markiert. In Differenzierungsstadien wird kein Histidin mehr sezerniert. Kapselsekret und Stilette bleiben bei diesen unmarkiert. Erst nach drei Tagen, wenn Sekretionsstadien mit einem hohen H3-Histidingehalt in die Differenzierungsphase eingetreten sind, erscheinen auch späte Differenzierungsstadien, bei denen sowohl das Kapselsekret als auch die Stilette 
stark markiert sind. Dies beweist, daß das Histidin nicht während der Differenzierung der Stilette im Plasma des Cnidoblasten gebildet, sondern vorher im Zentralsekret deponiert wird. Das gleiche gilt sicher auch für die übrigen Bausteine des Stilettproteins.

Damit sind zwei Funktionen des Zentralsekrets aufgezeigt: Es sorgt für die funktionsgerechte Lage des Nesselfadens in der Kapsel und ist zugleich Materialdepot für den Aufbau der Stilette.

\section{DISKUSSION}

In den elektronenmikroskopischen Arbeiten wird dem Zentralsekret nur wenig Beachtung geschenkt, obgleich es sich oft deutlich rom peripheren Kapselinhalt abhebt (Slau'tTerback \& Fawcett 1959, Slautterback 1963). WestFall (1966) weist auf die Entstehung des Nesselfadens in einer zentralen Anhäufung von Granula bei den basitrichen Haplonemen von Obelia hin. Aussagen uber Einzelheiten dieses Vorgangs kann die Autorin jedoch nicht machen. Sie neigt auf Grund der Untersuchungen an Metridium und Obelia zur Auffassung, daß der Faden in der Matrix der Kapselanlage allein oder auch in der Matrix des Außenschlauches entsteht, und deshalb höchstens der extracapsuläre Fadenteil in die Kapsel hinein verlagert werden muß. Die elektronenmikroskopischen Aufnahmen können aber die Möglichkeit nicht ausschließen, daß der Nesselfaden vollständig im Außenschlauch angelegt wird, wie der Befund bei Dipurena reesi nahelegt.

Interessant ist ein Vergleich der an $D$. reesi gewonnenen Ergebnisse mit den elektronenmikroskopischen Befunden von LoM \& VAvRA (1965) und Lom (1969). Die Autoren fanden zwischen den Polkapseln der Cnidosporidier und den Nesselkapseln der Cnidarier eine verblüffende Ubereinstimmung in Bau und Entwicklung. Sie stellten dabei auch fest, daß der Polfaden zuerst in einem mit der Kapselanlage in Verbindung stehenden Schlauch erscheint. Die Autoren schließen jedoch nicht aus, daß der Polfaden z. T. auch in der Kapselanlage gebildet wird.

Auch das Zentralsekret scheint in Form der "course granules" (Lom \& VAVRA 1965 ) und der „electron dense globules" (Lom 1969) vorhanden zu sein. Die zentrale Lage dieser Substanz ist auf den Bildern oft gut zu erkennen, und Abbildung 10 von LoM \& VAVRA läßt kaum noch einen $Z$ weifel darüber, daß auch hier dieses "Zentralsekret" als Spule für den einwandernden Faden dient.

Die Entstehung des Schaftes mit seiner Bewehrung in der für Dipurena reesi beschriebenen Weise wurde bei anderen Arten noch nicht sicher festgestellt. Aber schon WiLl (1910) hat auf Grund seiner Beobachtungen an Hydra und Syncoryne vermutet, daß die Schaftwand der Stenotelen vom Offnungspol der Kapselanlage her über die Stilettanlagen wächst, und CHAPMAN \& TrLNEY (1959) balten ebenfalls eine Entstehung der Schaftwand in dieser Weise bei den Stenotelen von Hydra für wahrscheinlich. Allerdings fanden die Autoren Kapselanlagen mit vollständiger Schaftwand, aber ohne Stilette und Dornen. Sie schließen daraus, daß die Bewehrung erst nach der Ausdifferenzierung der Schaftwand gebildet wird. CHAPMAN \& TrLNEY nehmen daher auch an, $\mathrm{daB}$ die Stilette und Dornen an der Schaftwand entstehen, mit dieser also von Anfang an fest verbunden sind, während ja bei $D$. reesi diese Verbindung erst im Lauf des Wachstums der Schaftwand hergestellt wird. Wie dies geschieht, bleibt allerdings noch unklar. 


\section{ZUSAMMENFASSUNG}

1. Histochemische und autoradiographische Untersuchungen ïber die Entstehung des Nesselschlauchs in den Nematocysten des Hydroidpolypen Dipurena reesi VANUCCI haben gezeigt, daß das Zentralsekret der Kapselanlage für die funktionsgerechte Lage des Nesselfadens in der Kapsel sorgt. Die Entstehung des Nesselfadens außerhalb der Kapselanlage ist sehr wahrscheinlich.

2. Das Zentralsekret hat weiterhin die Funktion eines Materialspeichers. Es löst sich nach der Aufspulung des Nesselfadens im peripheren Kaspelinhalt auf. Das im Zentralsekret nachgewiesene Histidin gelangt in die Stilettanlagen.

3. Die Stilette und Borsten des Schaftes werden auf einem PJS-positiven Achsenfaden angelegt. Die Schaftwand umwächst vom Apikalpol der Kapselanlage her die Stilett- und Borstenanlagen.

\section{ZITIERTE LITERATUR}

Brinkmann-Voss, A., 1970. Anthomedusae, Athecatae (Hydrozoa, Cnidaria) of the Mediterranean. P. 1: Capitata. Fauna Flora Golfo Napoli. 39, 1-96.

Chapman, G. B. \& Truney, L. G., 1959. Cytological studies of the nematocysts of Hydra. II. The stenoteles. J. biophys. biochem. Cytol. 5, 79-84.

EWALD, A., 1915. Uber den Bau, die Entladung und die Entwicklung der Nesselkapsehn von Hydra und Porpita mediterranea nebst einigen histologischen Bemerkungen uber die letztere Form. Verh. naturh.-med. Ver. Heidelb. N. F. 13, 303-354.

GüNZL, H., 1964. Untersuchungen über die Auslösung der Medusenknospung bei Hydroidpolypen. Zool. Jb. (Anat. Ontogenie Tiere) 81, 491-528.

- 1968. Uber die Reifung der Nesselkapseln bei Dipurena reesi Vannucci (Hydrozoa). Z. Zellforsch. mikrosk. Anat. 89, 509-518.

IWANZorf, N., 1896. Uber den Bau, die Wirkungsweise und die Entwicklung der Nesselkapseln bei Coelenteraten. Bull. Soc. Impr. Nat. Mosc. N. F. 10, 323-355.

JiCKelI, C. F., 1883. Der Bau der Hydrödpolypen. I. Uber den histologischen Bau von Eudendrium EHRBG. und Hydra L. Morph. Jb. 8, 373-416.

Lom, J., 1969. Notes on the ultrastructure and sporoblast development in fish parasitizing myxosporidian of the genus Sphaeromyxa. Z. Zellforsch. mikrosk. Anat. 97, 416-437.

- \& VAVRA, J., 1965. Notes on the morphogenesis of the polar filament in Henneguya (Protozoa, Cnidosporidia). Acta protozool. 3, 57-60.

Möвrus, K., 1866. Über den Bau, den Mechanismus und die Entwidklung der Nesselliapseln einiger Polypen und Quallen. Abh. naturw. Ver. Hamburg 5, 1-15.

Romexs, B., 1948. Mikroskopische Technik. Oldenbourg, Müinchen. 695 pp.

Schnerder, K. C., 1900. Mitteilungen über Siphonophoren. V. Nesselzellen. Arb. zool. Inst. Univ. Wien 12, 133-242.

Slautterback, D. B., 1963. Cytoplasmic microtubules. I. Hydra. J. Cell Biol. 18, 367-388.

- \& FA WCETT, D. W., 1959. The development of the cnidoblasts of Hydra. J. biophys. biochem. Cytol. 5, 441-452.

Spannizof, L., 1967. Einfuihrung in die Praxis der Histochemie, VEB Fischer, Jena, 172 pp.

WESTFALL, J. A., 1966. The differentiation of nematocysts and associated structures in the Cnidaria. Z. Zellforsch. mikrosk. Anat. 75, 381-403.

WILL, L., 1910. Die sekretorischen Vorgänge bei der Nesselkapselbildung der Coelenteraten. Sber: Abli.haturf. Ges. Rostock. N. F. 2, 1-41.

Anschrift des Autors: Dr: H. GüNzix

Zoologisches:Institut der Universität

7,400 Tübingent

Hölderlinstraße 12

Bundesrepublik Deutschland 